\title{
Influence of Vertical Mouth Opening on Oral Appliance Treatment Outcome in Positional Obstructive Sleep Apnea
}

Francesca Milano, DMD,2; Sabrina Mutinelli, DMD1,3; Kate Sutherland, PhD 4,5; Giulia Milioli, MD²; Giuseppe Scaramuzzino, MD²; Antonella Bacchieri Cortesi, DMD'; Giuseppe Siciliani, DDS'1; Luca Lombardo, DMD'; Peter Cistulli, MD, PhD ${ }^{4,5}$

${ }^{1}$ Postgraduate School of Orthodontics, University of Ferrara, Ferrara, Italy; ${ }^{2}$ Sleep Clinic, Clinica Privata Villaba, GVM, Bologna, Italy; ${ }^{3}$ LUdeS

Foundation Higher Education Institution, Malta; ${ }^{4}$ Department of Respiratory and Sleep Medicine, Royal North Shore Hospital, Sydney, New

South Wales, Australia; ${ }^{5}$ Charles Perkins Centre, Sydney Medical School, University of Sydney, Sydney, New South Wales, Australia

\begin{abstract}
Study Овjectives: The supine sleeping posture can heighten the risk of sleep-disordered breathing events. Patients with positional obstructive sleep apnea (OSA) are characterized by a supine apnea-hypopnea index (AHI) that is at least two times higher than in nonsupine positions. This study aimed to assess the influence of mouth opening on the outcome of mandibular advancement splint (MAS) treatment in patients with positional OSA.

MethoDs: The secondary data of 230 individuals treated for positional OSA with MAS (standard MAS group) or with MAS plus vertical elastics to prevent mouth opening (MAS+elastics group) were compared in terms of treatment response. Treatment success was defined as at least a 75\% reduction in AHI from baseline. Secondary outcomes included the change in AHI, supine AHI, and nonsupine AHI.

RESULTs: Both groups showed a significant improvement in AHI with MAS in situ. The improvement in AHI, supine AHI, and nonsupine AHI with MAS was significantly greater in the MAS+elastics group than in the standard MAS group. Treatment success rate was significantly higher in the MAS+elastics group $(67.4 \%$ versus $36.2 \% ; P<.001)$. After adjusting for potential confounders, the odds of successful treatment increased 3.8-fold through the use of vertical elastics.

Conclusions: This pilot study suggests that vertical elastics that minimize mouth opening enhance the outcome of MAS treatment in patients with positional OSA. These findings support the need for further research to verify the role of vertical elastics with bimaxillary oral appliances to improve the response to treatment in patients with positional OSA.
\end{abstract}

KEYWORDS: mandibular advancement splints, mouth opening control, positional OSA, vertical opening,

Citation: Milano F, Mutinelli S, Sutherland K, Milioli G, Scaramuzzino G, Cortesi AB, Siciliani G, Lombardo L, Cistulli P. Influence of vertical mouth opening on oral appliance treatment outcome in positional obstructive sleep apnea. Journal of Dental Sleep Medicine. 2018;5(1):17-23.

\section{INTRODUCTION}

Obstructive sleep apnea (OSA) is a respiratory sleep-related disorder, characterized by repetitive complete (apneas) and/ or partial (hypopneas) airways collapse. The diagnosis and severity of OSA is generally measured by the apnea-hypopnea index (AHI), which represents the number of obstructive events per hour of sleep, after correlation with clinical history and examination. Patients affected by positional OSA are characterized by an AHI in the supine position (supine AHI) that is at least two times higher than in nonsupine positions (nonsupine AHI). ${ }^{1}$ Furthermore, some patients with positional OSA are only affected in the supine position, termed supineisolated OSA, and defined by a supine AHI that is at least two times higher than nonsupine AHI, and a nonsupine AHI $<5$ events/h (no OSA). These patients appear to represent a distinct phenotype that may be particularly responsive to certain OSA therapies, including positional therapy ${ }^{2}$ and mandibular advancement splint (MAS) therapy. ${ }^{3}$

An MAS is an oral appliance that is worn to protrude the mandible during sleep, thereby increasing upper airway dimensions, reducing collapsibility, and decreasing or eliminating the respiratory events during sleep. A number of previous studies have reported a higher response to MAS treatment in patients with positional OSA..$^{3-6}$ In contrast, a large study by Sutherland et al. ${ }^{7}$ did not find positional OSA to be a predictor of treatment response. A potentially important difference among these studies was the device characteristics. A one-piece device ${ }^{3}$ or a two-piece device with a little freedom for vertical opening ${ }^{8}$ were found to be effective in positional OSA. However, two-piece appliance designs that allow for complete opening of the mandible appear to show a decreased response rate in positional OSA. ${ }^{7}$ Mouth opening increases upper airway collapsibility, increasing critical closing pressure (Pcrit). Two studies ${ }^{9,10}$ have demonstrated that appliances that control mouth opening are more effective in decreasing AHI. In contrast, a two-piece device is generally more comfortable than a one-piece appliance, because the patient is not completely restricted and mandibular movements are allowed, and this has a positive effect on compliance. ${ }^{11}$ Hence, it is important to clarify the role of mouth opening on the efficacy of MAS treatment, particularly in positional OSA.

Accordingly, the aim of this retrospective study was to evaluate whether minimization of mouth opening with vertical elastics enhances the effectiveness of treatment with a twopiece MAS in patients with positional OSA. 


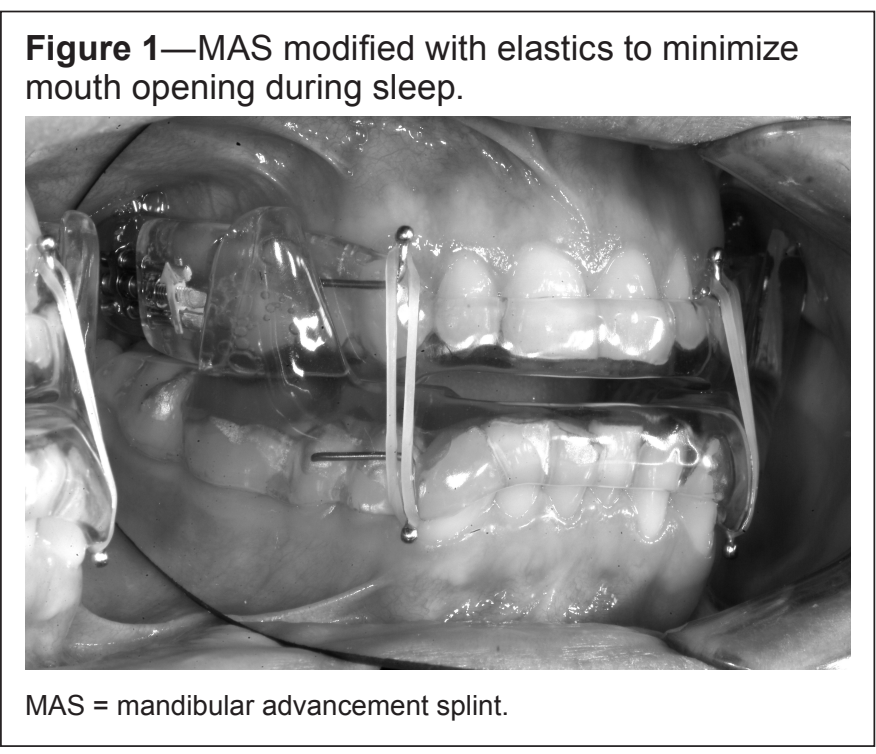

\section{METHODS}

This was a retrospective study using data from positional OSA cases treated at two centers with differing approaches to the use of vertical elastics to control mouth opening. The total sample comprised 230 individuals, 188 males (mean age, 51.1 years, standard deviation [SD] 11.8) and 42 females (mean age, 55.2 years, SD 8.9) treated with a MAS for positional OSA. One hundred thirty-eight patients (105 males and 33 females) were treated in sleep centers in Sydney, Australia in the years from 2000 to 2013 and 92 patients (83 males and 9 females) at the Orthodontic Department of the University of Ferrara, Italy in the time period between 2012 and 2015.

All patients had positional OSA (supine-predominant or supine-isolated OSA), confirmed with either polygraphy (Embletta, Natus Medical Incorporated) or full polysomnography (Compumedics Ltd, Australia), according to the diagnostic criteria of The AASM Manual for the Scoring of Sleep and Associated Events: Rules, Terminology and Technical Specifications. ${ }^{12}$ All patients presented with at least two of the following symptoms: snoring, fragmented sleep, witnessed apneas, and daytime sleepiness. Body mass index (BMI) was also recorded. For each patient a report of a six-channel polygraphic home sleep test or in-laboratory polysomnography was analyzed to record the mean $\mathrm{AHI}$, supine $\mathrm{AHI}$, nonsupine $\mathrm{AHI}$ at baseline, and with MAS in situ (after 4 to 6 months acclimatization).

In order to account for differences between diagnostic study types (polygraphy [PG] and polysomnography [PSG]), the primary analysis was focused on the AHI percentage change obtained with MAS in situ.

In addition, to evaluate the actual OSA severity of patients monitored with PG, baseline AHI indices were subsequently adjusted using the method of Dingli et al..$^{13}$ To apply this adjustment method, patients diagnosed with PG were first organized into the following groups: OSA (AHI $\geq 20$ events/h), possible OSA (AHI $\geq 10$ and $<20$ events/h), or not OSA (AHI $<10$ events/h). Next, the appropriate correction was applied to the AHI scores in each group. Average percentage AHI difference between PSG and PG estimates were added to the portable device AHI scores to account for the underestimation based on total study time. The portable device AHI scores were thus increased by $44 \%$ for the not OSA group, $30 \%$ for the possible OSA group, and $13 \%$ for the OSA group.

\section{Appliance Description}

All patients, across both sites, were treated exclusively with a customized two-piece MAS device (SomnoDent MAD, SomnoMed Ltd, Australia). The Sydney, Australia site (standard MAS group) employed a traditional MAS. In contrast, the Ferrara, Italy site employed a MAS that was modified with elastics to minimize mouth opening during sleep (MAS+elastics group). This construct is demonstrated in Figure 1. For the MAS+elastics group the MAS was constructed with hooks for vertical elastic positioning and patients were instructed to wear the elastics and to replace them once a week. Vertical elastics were carefully selected not to interfere with device retention, to avoid the possibility of dislodging one of the two pieces of the MAS at the maximal mouth opening (range 85-170 gr, $\left.3 / 83 / 16^{\prime \prime}\right)$. Patients in the standard MAS group simply used the device without vertical elastics. Vertical anterior opening was kept to a minimum (approximately $5 \mathrm{~mm}$ ) in both groups. Similar titration protocols were used at both sites (ie, the device was incrementally titrated to the maximal comfortable limit of advancement over a 4 - to 8 -week period).

Patients were asked to incrementally titrate the appliance as far forward as comfortably possible. In case of temporomandibular joint pain or discomfort the advancement was stopped or reduced to achieve the most comfortable position.

\section{Treatment Response}

The treatment response (primary outcome) to type of appliance (standard versus modified with elastics) was defined by measuring the percentage AHI reduction with MAS in situ from baseline and defining the cutoff between responders (success) and nonresponders (failure) at $75 \%$. We chose this definition to align with the guidelines published in 2007 by the American Academy of Sleep Medicine. ${ }^{14}$ They defined an adequate titration as "one that does reduce the overnight AHI by $75 \%$ from baseline (especially in severe patients)."

Secondary outcomes analyzed were: absolute and percentage change in AHI, and change in supine AHI and nonsupine AHI.

\section{Statistical Analysis}

Baseline characteristics of the sample were compared (age, sex distribution, BMI, AHI, supine AHI, nonsupine AHI, and OSA phenotype) to assess the homogeneity between groups.

Between-group comparisons were performed using the $t$ test and the Wilcoxon rank-sum test for independent normally and non-normally distributed data (Shapiro-Wilk test, $a$-level $P<.05)$ of continuous variables and the Pearson $\chi^{2}$ test or the Fisher exact test in case of categorical variables.

Within each of the two groups, the change in PG or PSG variables (AHI, supine AHI, and nonsupine AHI) with MAS in situ were analyzed using the Wilcoxon signed-rank test due to non-normal distribution of the variables (Shapiro-Wilk test, $P<.001)$. To evaluate the effect of MAS with and without elastics (MAS+elastics group versus standard MAS group), a 


\begin{tabular}{|c|c|c|c|}
\hline & Standard MAS & MAS+Elastics & $P$ \\
\hline Full Sample & $n=138$ & $\mathrm{n}=92$ & \\
\hline$\%$ males $/ \%$ females & $76.1 / 23.9$ & $90.2 / 9.8$ & $.007^{\mathrm{a}}$ \\
\hline Age, years, mean (SD) & $50.8(10.6)$ & $53.4(12.4)$ & $.097^{\mathrm{b}}$ \\
\hline AHI, median (IQR) & $20.9(15.0)$ & $27.0(16.7)$ & $.0004^{\mathrm{c}}$ \\
\hline Supine $\mathrm{AHI}$, median (IQR) & $41.6(35.8)$ & $45.8(25.5)$ & $.3003^{c}$ \\
\hline Nonsupine AHI, median (IQR) & $7.0(11.4)$ & $11.4(14.0)$ & $.0002^{\mathrm{c}}$ \\
\hline $\begin{array}{l}\text { Phenotype }^{d} \\
\text { Supine-predominant OSA, n (\%) } \\
\text { Supine-isolated OSA, n (\%) }\end{array}$ & $\begin{array}{l}83.0(60.1) \\
55.0(39.9)\end{array}$ & $\begin{array}{l}76.0(82.6) \\
16.0(17.4)\end{array}$ & \\
\hline BMI, kg/m², median (IQR) & $27.6(6.5)$ & $26.5(4.0)$ & $.0097^{\mathrm{c}}$ \\
\hline Males & $n=105$ & $\mathrm{n}=83$ & \\
\hline Age, years, mean (SD) & $49.7(10.9)$ & $52.9(12.6)$ & $.0693^{\mathrm{b}}$ \\
\hline AHI, median (IQR) & $21.4(15.4)$ & $27.0(17.3)$ & $.0213^{c}$ \\
\hline $\begin{array}{l}\text { AHI severity distribution } \\
\text { Mild OSA, } n(\%) \\
\text { Moderate OSA, n (\%) } \\
\text { Severe OSA, n (\%) }\end{array}$ & $\begin{array}{l}18.0(17.1) \\
57.0(54.3) \\
30.0(28.6)\end{array}$ & $\begin{array}{r}9.0(10.9) \\
39.0(47.0) \\
35.0(42.1)\end{array}$ & \\
\hline BMI, kg/m², median (IQR) & $27.6(5.9)$ & $26.6(4.0)$ & $.0086^{c}$ \\
\hline Females & $n=33$ & $\mathrm{n}=\mathbf{9}$ & \\
\hline Age, years, mean (SD) & $54.3(8.5)$ & $58.4(10.0)$ & $.2862^{\mathrm{b}}$ \\
\hline AHI, median (IQR) & $17.1(12.9)$ & $27.0(20)$ & $.0055^{c}$ \\
\hline $\begin{array}{l}\text { AHI severity distribution }{ }^{f} \\
\text { Mild OSA, n (\%) } \\
\text { Moderate OSA, n (\%) } \\
\text { Severe OSA, n (\%) }\end{array}$ & $\begin{array}{r}14.0(42.4) \\
14.0(42.4) \\
5.0(15.2)\end{array}$ & $\begin{array}{c}- \\
5.0(55.6) \\
4.0(44.4)\end{array}$ & \\
\hline BMI, kg/m², median (IQR) & $27.8(7.6)$ & $26.0(8.0)$ & $.9874^{\mathrm{c}}$ \\
\hline
\end{tabular}

Mild OSA defined as AHI $\geq 5$ to $<15$ events/h. Moderate OSA defined as AHI $\geq 15$ to $\leq 30$ events/h. Severe OSA defined as AHI $>30$ events/h. $\mathrm{a}=$ Pearson $X^{2}$ test or Fisher exact test. $\mathrm{b}=t$ test for independent data with unequal variance. $c=$ two-sample Wilcoxon rank-sum test. $\mathrm{d}=$ Pearson $\mathrm{X}^{2}$ test, $P<.001$. e $=$ Pearson $\mathrm{X}^{2}$ test, $P=.120 \mathrm{f}=$ Fisher exact test, $P=.031$. AHI $=$ apnea-hypopnea index, $\mathrm{BMI}=$ body mass index, IQR = interquartile range, MAS = mandibular advancement splint, OSA = obstructive sleep apnea, SD = standard deviation.

dichotomous variable "treatment response" was generated designating patients as "responders" (at least a 75\% of AHI reduction) or "nonresponders" (AHI reduction below 75\%). The proportions of responders in each group (MAS+elastics group and standard MAS group) were compared using the Pearson $\chi^{2}$ test. To compare treatment effectiveness between groups, the percentage reduction in AHI from baseline was analyzed using the two-sample Wilcoxon rank-sum test (Shapiro-Wilk test, $P<.001)$.

A logistic regression model was built (step-up procedure) to control for potential confounders (baseline AHI, age, sex, BMI, and OSA phenotype), which could be associated with treatment response and modify the effect of the oral appliance.

All data were statistically analyzed with the software package STATA 14.1 (StataCorp LP, College Station, Texas, United States).

\section{Sample Size Estimation}

To detect a $20 \%$ difference in proportion of positive treatment response between standard MAS group and MAS+elastics group, a sample size of 186 patients (93 patients per group) is required (Pearson $\chi^{2}$ test; proportion $1=0.5$; proportion $2=0.7$; $\alpha$-level $=0.05$; power $=0.80$ ).

\section{RESULTS}

\section{Difference at Baseline Between the Two Groups}

A comparison of the baseline characteristics of the standard MAS group and MAS+elastics group, for the total sample and sex subgroups, is shown in Table 1.

The median AHI was higher in the MAS+elastics group than in the standard MAS (two-sample Wilcoxon rank-sum test, $P=.0004)$. The men presented a similar OSA severity in the MAS+elastics group and in the standard MAS (moderate to severe OSA occurring in $89.2 \%$ versus $82.9 \%$ [ $\chi^{2}$ test, $P=.120$ ], respectively) (Table 1). In contrast, all females of the MAS+elastics group (100\%) suffered from moderate or severe OSA versus $57.6 \%$ in the standard MAS group (Fisher exact test, $P=.031$ ). It must be noted that the sample size of MAS+elastics group was small with only nine females.

In regard to the underestimation of AHI from portable devices, after having adjusted the values using the method of Dingli et al., ${ }^{13}$ in the MAS+elastics group 28 patients (30.4\%) were in the possible OSA group and 64 patients $(69.6 \%)$ were in the OSA group. After that, all AHI measurements at baseline were increased by the corresponding percentage. Adjusted and unadjusted AHI values are listed separately in Table 2 . The 
Table 2-Baseline descriptive statistics of unadjusted and adjusted $\mathrm{AHI}$ in MAS+elastics group.

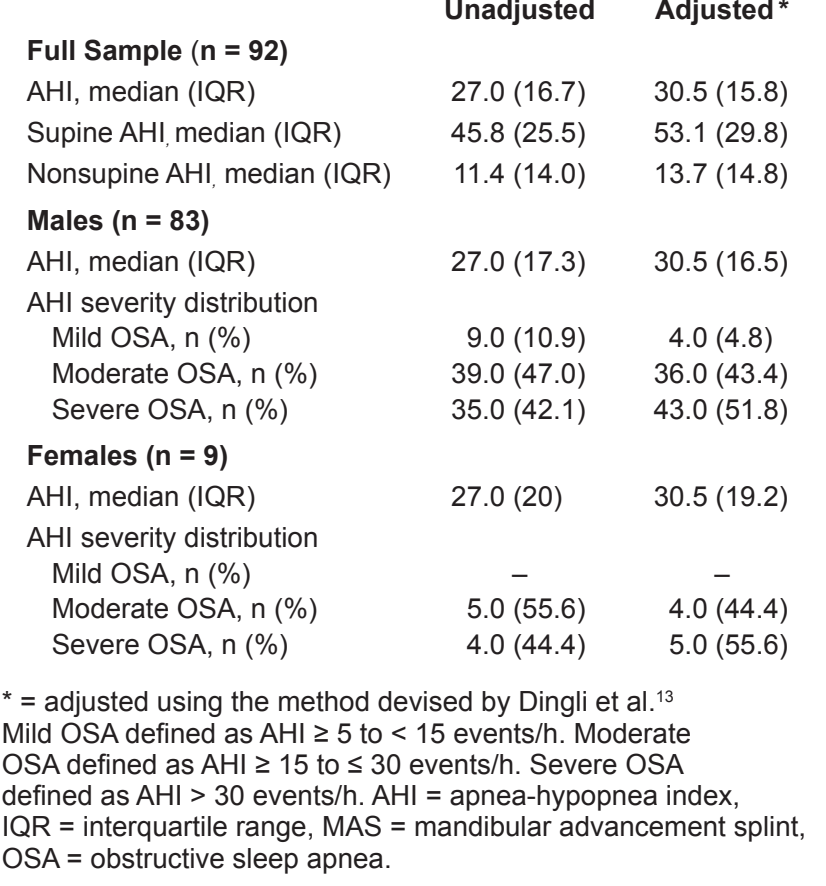

comparison of OSA severity distribution between standard MAS and adjusted MAS+elastics group showed a more severe OSA in both men ( $\chi^{2}$ test, $\left.P=.001\right)$ and women (Fisher exact test, $P=.012)$ of the second group.

There was no difference in age between the two groups (twosample $t$ test, $P=.097$ ) (Table 1) either in the whole group or by sex.

The ratio of males to females was different between groups: $76.1 \%$ of males and $23.9 \%$ of females in the standard MAS group versus $90.2 \%$ of males and $9.8 \%$ of females in the MAS+elastics group ( $\chi^{2}$ test, $P=.007$ ).

Median BMI showed a significant difference between males of the two groups. There were no severe or morbidly obese patients in the MAS+elastics group whereas there were in the standard MAS group: $35.5 \%$ of the male patients were obese $(\mathrm{BMI} \geq 30)$ in the standard MAS group and in $15.2 \%$ in the MAS+elastics group (Pearson $\chi^{2}$ test, $P=.001$ ).

BMI median value was not different between females of the standard MAS group and the MAS+elastics group. However, there was a relatively small number of females in the sample.

The position phenotypes of supine-predominant versus supine-isolated OSA was unbalanced between the groups (Pearson $\chi^{2}$ test, $P<.001$ ). In the MAS+elastics group, the percentage of patients with supine-predominant OSA was higher than in the standard MAS group (82.6\% versus $60.1 \%$ ). Conversely, the percentage of patients with supine-isolated OSA was lower in the MAS+elastics group than in the standard MAS group (17.4\% versus 39.9\%).

\section{Comparison Between Groups}

The reduction in AHI, supine AHI, and nonsupine AHI with MAS in situ was significantly higher in the MAS+elastics group than in the standard MAS group (two-sample Wilcoxon rank-sum test, $P<.001$ ) as summarized in Table 3. There was a higher percentage of treatment responders in the MAS+elastics group (67.4\%) than in the standard MAS group (36.2\%) (Pearson $\chi^{2}$ test, $P<.001$ ) (Table 3 and Figure 2).

When analyzing the within-group changes, both groups showed an improvement in AHI with MAS in situ (Table 3). The percentage reduction in AHI from baseline (treatment effectiveness) reached a median value of $64 \%$ (interquartile range 0.5 ) in the standard MAS group (Wilcoxon signedrank test, $P<.001$ ) and $81 \%$ (interquartile range 0.2 ) in the MAS+elastics group (Wilcoxon signed-rank test, $P<.001$ ). Improvement in AHI, supine AHI, and nonsupine AHI with MAS in situ was also evident (Wilcoxon signed-rank test, $P<.001)$ in both groups. Descriptive statistics for each variable has been reported in Table 3. Position-dependent OSA was still present in the standard MAS group (47.7\% nonresponders) and in the MAS+elastics group (46.6\% nonresponders).

A logistic regression model was built to look at the influence of the addition of elastics to MAS on treatment response while controlling for other factors (age, sex, OSA severity, and BMI) known to influence treatment response. OSA phenotype (supine-predominant or supine-isolated positional OSA), supine $\mathrm{AHI}$, and nonsupine AHI were also considered in the model.

The exposure selection was performed step-forward, after having graphically evaluated the trend of the points to check the linearity assumption. Age $(P=.017)$, BMI $(P=.016)$, and baseline $\mathrm{AHI}$ influenced the odds ratio (OR) in the two groups analyzed. More specifically the covariate baseline AHI did not show a linear trend of the points, as observed for the other exposures. The shape was a curve with an increase in response to treatment followed by a reduction as baseline AHI increased (reverse U-shape), which was best described by means of a quadratic polynomial $(P=.008)$.

The Mantel-Haenszel test and test of homogeneity were performed to confirm if OSA severity and BMI were confounders and could modify the association between MAS treatment type and the response to treatment. The significant output of the Mantel-Haenszel test $(P<.001)$ confirmed that the two exposures were confounders. The exposure sex was kept in the model $(P=.877)$ because we were specifically interested in testing its influence on the outcome. The covariate OSA phenotype (supine-predominant or supine-isolated OSA) did not reach an adequate level of significance $(P=.113)$ as supine AHI $(P=.992)$ variable registered at baseline. Nonsupine AHI $(P=.010)$ was at first selected and then dropped because it did not reach significance in the complete model. Consequently, these three variables were not included. No significant interactions between exposures were noted.

The equation of the final model, which assessed the effect of type of appliance (exposure group) on the odds of a positive response to treatment after controlling for the other covariates, was the following:

$\log \left(\right.$ Odd $\left._{i}\right)=1.394+1.333^{\star} \operatorname{group}_{i}+0.891^{\star}$ sex $_{i}-0.050^{\star}$ age $0.090^{\star} \mathrm{BMI}+0.117^{\star} \mathrm{AHI}-0.001^{\star} \mathrm{AHI}{ }^{2}$

Model fitting was evaluated by receiver operating characteristic curve and estimating the area under it (Figure 3). The 
goodness of fit was 0.76 , which is consistent with an acceptable discrimination.

Coefficients and OR for each covariate and statistics output are reported in Table 4.

The model shows a strong influence of MAS modified with elastics and a positive response to treatment compared with the standard MAS group (OR 3.8, $P<.001$ ) while controlling for the other variables influencing MAS response.

Figure 2-Treatment response prevalence in the two groups estimated as the percentage of $\mathrm{AHI}$ reduction with the MAS from the baseline.
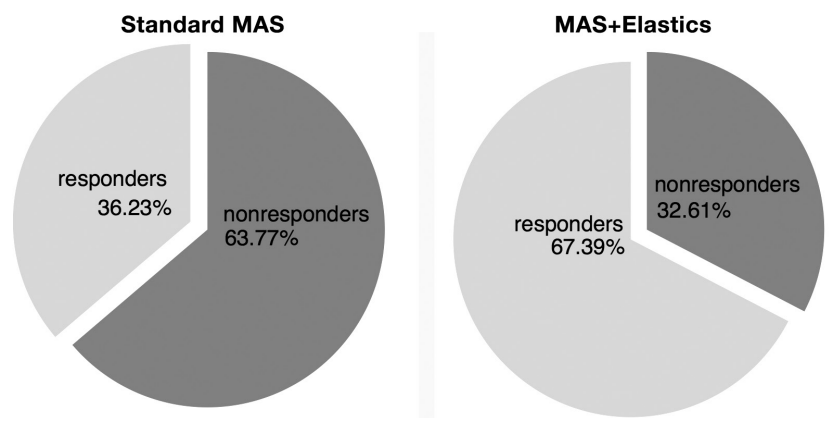

$\mathrm{AHI}=$ apnea-hypopnea index, MAS = mandibular advancement splint.
Female sex has a positive influence on treatment outcome (OR 2.4, $P=.030$ ). In contrast, for each unit increase in age or BMI the odds to be a responder were reduced, by $5 \%$ and $9 \%$, respectively. Also, baseline AHI was associated with treatment outcome following a curvilinear trend: in mild and moderate

Figure 3-Multiple logistic regression model fitting displayed by means of ROC curve.

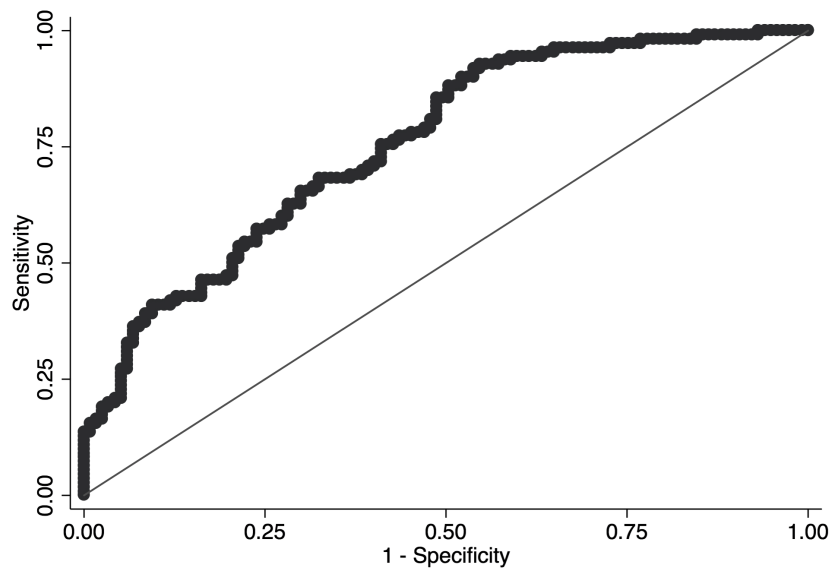

The model describes the response to treatment of the sample with five explanatory variables (type of appliance, sex, age, body mass index, and baseline apnea-hypopnea index). Area under ROC curve $=0.7556$. $R O C=$ receiver operating characteristic.

Table 3-Treatment effects of MAS in the standard MAS and MAS+elastics group.

\begin{tabular}{|c|c|c|c|c|c|}
\hline & $\begin{array}{c}\text { Standard MAS } \\
\quad(n=138)\end{array}$ & $\begin{array}{c}\text { Change Within } \\
\text { Group, } P\end{array}$ & $\begin{array}{l}\text { MAS+Elastics } \\
\quad(n=92)\end{array}$ & $\begin{array}{l}\text { Change Within } \\
\text { Group, } P\end{array}$ & $\begin{array}{c}\text { Comparison } \\
\text { Between Groups, } P \\
\end{array}$ \\
\hline \multicolumn{6}{|l|}{ AHI with MAS } \\
\hline Median (IQR) & $8.2(12.5)$ & & $5.0(6.0)$ & & \\
\hline Range & 0 to 60 & & 0 to 36 & & \\
\hline $\begin{array}{l}\text { AHI change } \\
\text { Median (IQR) } \\
\text { Range }\end{array}$ & $\begin{array}{l}-12.4(15.8) \\
-57.3 \text { to } 30.0\end{array}$ & $<.001^{\mathrm{a}}$ & $\begin{array}{l}-21.7(15.0) \\
-60.0 \text { to }-1.4\end{array}$ & $<.001^{\mathrm{a}}$ & $<.001^{b}$ \\
\hline \multicolumn{6}{|l|}{ Supine AHI with MAS } \\
\hline Median (IQR) & $14.3(20.1)$ & & $7.0(9.4)$ & & \\
\hline Range & 0.7 to 94.1 & & 0 to 60 & & \\
\hline $\begin{array}{l}\text { Supine AHI change } \\
\text { Median (IQR) } \\
\text { Range }\end{array}$ & $\begin{array}{l}-22.4(26.7) \\
-91.3 \text { to } 69.8\end{array}$ & $<.001^{\mathrm{a}}$ & $\begin{array}{c}-35.7(23.6) \\
-110.0 \text { to }-4.0\end{array}$ & $<.001^{\mathrm{a}}$ & $<.001^{\mathrm{b}}$ \\
\hline \multicolumn{6}{|c|}{ Nonsupine AHI with MAS } \\
\hline Median (IQR) & $4.2(6.6)$ & & $2.0(4.9)$ & & \\
\hline Range & 0 to 33.5 & & 0 to 19 & & \\
\hline $\begin{array}{l}\text { Nonsupine } \mathrm{AHI} \text { change } \\
\text { Median (IQR) } \\
\text { Range }\end{array}$ & $\begin{array}{c}-2.2(10.4) \\
-43.1 \text { to } 19.3\end{array}$ & $<.001^{\mathrm{a}}$ & $\begin{array}{l}-8.5(10.5) \\
-30 \text { to } 4\end{array}$ & $<.001^{\mathrm{a}}$ & $<.001^{b}$ \\
\hline \multicolumn{6}{|c|}{ Treatment effectiveness $^{c}$} \\
\hline Median (IQR) & $0.64(0.52)$ & & $0.81(0.20)$ & & \\
\hline Range & -1.4 to 1.0 & $<.001^{\mathrm{a}}$ & 0.1 to 1.0 & $<.001^{\mathrm{a}}$ & $<.001^{\mathrm{b}}$ \\
\hline \multicolumn{6}{|l|}{ Treatment response $^{d}$} \\
\hline Responders, n (\%) & $50(36.2)$ & & $62(67.4)$ & & $<.001^{\mathrm{e}}$ \\
\hline Nonresponders, n (\%) & $88(63.8)$ & & $30(32.6)$ & & \\
\hline
\end{tabular}

$a=$ Wilcoxon signed-rank test. $b=$ Two-sample Wilcoxon rank-sum test. $c=$ Proportion of reduction in AHI from baseline. $d=$ Positive treatment response (responders) is defined when AHI reduction with MAS in situ is at least a $75 \%$ from baseline. $e=P e a r s o n ~ X^{2}$ test. AHI $=$ apneahypopnea index, IQR = interquartile range, MAS = mandibular advancement splint. 
Table 4-Multiple logistic regression model of response to treatment (responders versus nonresponders) with five explanatory variables (type of appliance, sex, age, BMI, and baseline AHI).

\begin{tabular}{|c|c|c|c|c|c|}
\hline Variable & Coefficient (ß) & Standard Error & Z Test & $P$ & Odds Ratio $(95 \% \mathrm{Cl})$ \\
\hline Intercept & 1.394 & 1.384 & & & \\
\hline Group $^{a}$ & 1.333 & 0.330 & 4.04 & $<.001$ & 3.794 (1.988 to 7.239$)$ \\
\hline Sex ${ }^{b}$ & 0.891 & 0.411 & 2.17 & .030 & 2.437 (1.090 to 5.449$)$ \\
\hline Age in years & -0.050 & 0.014 & -3.51 & $<.001$ & 0.952 (0.926 to 0.978$)$ \\
\hline BMI & -0.090 & 0.040 & -2.27 & .023 & 0.914 (0.845 to 0.988$)$ \\
\hline Baseline $\mathrm{AHI}$ & 0.117 & 0.038 & 3.03 & .002 & $1.124(1.042$ to 1.212$)$ \\
\hline $\mathrm{AHI}$ & -0.001 & 0.001 & -2.29 & .022 & $0.999(0.998$ to 1.000$)$ \\
\hline
\end{tabular}

$\mathrm{a}=$ Reference group is control standard MAS group. $\mathrm{b}=$ Reference group is men. $\mathrm{AHI}=$ apnea-hypopnea index, $\mathrm{BMI}=$ body mass index, $\mathrm{Cl}=$ confidence interval.

OSA the positive effect of treatment increased as AHI increased, but in severe OSA there was a tendency to invert the trend.

\section{DISCUSSION}

Our retrospective study is the first evaluation on a large number of patients of the potential influence of mouth opening on MAS treatment outcome in patients with positional OSA. The results showed a significant decrease in $\mathrm{AHI}$, supine $\mathrm{AHI}$, and nonsupine $\mathrm{AHI}$ in both groups, but the percentage reduction in AHI from baseline and the proportion of responders reached a higher value in the group of patients treated with vertical elastics. Specifically, the use of vertical elastics to prevent mouth opening improved treatment success (final AHI reduction at least of $75 \%$ from the baseline AHI) in a clinically relevant way.

This finding extends recent research that has identified the importance of phenotypic characteristics on OSA treatment response by highlighting the potential importance of MAS design features in relation to specific phenotypes as part of a personalized approach to treatment. The role played by the body position in the genesis of obstructive respiratory events and on the response of patients with OSA to available treatments has been studied by many investigators, covering influences on respiratory mechanics, lung volume, and critical pressure, all reflecting an increase in upper airway collapsibility ${ }^{15-17}$ Among the several mechanisms that may contribute to airway collapse in the supine sleeping position, the force of gravity promotes tongue collapse and mandibular opening, the latter resulting in a downward rotation of the mandible and further facilitating the tongue collapse. Mandibular advancement devices are expected to improve the upper airway crosssectional area in the supine position, because of their effect on the protrusion of the mandible, and also for their capability to stabilize the mandible and counteract the effect of gravitation. ${ }^{18}$ The control of mandibular collapse in the supine position depends on mandibular advancement device design. Comparison of studies of patients with OSA treated with a one-piece or a two-piece device suggests differences in MAS treatment response in patients with positional OSA..$^{3,718}$ Marklund et al. ${ }^{3}$ and Sutherland et al. ${ }^{7}$ describe conflicting results regarding body position as a predictive positive factor on MAS treatment outcome. Marklund et $\mathrm{al}^{3}{ }^{3}$ concluded that supine-dependent OSA is a good predictive factor on MAS treatment. The device employed for the study was a Monobloc that prevents mouth opening during sleep. The same appliance (Monobloc) was used by Takaesu et al. ${ }^{18}$ who demonstrated that male patients with positional OSA can achieve a similar outcome with MAS or nasal continuous positive airway pressure treatment. However, Sutherland et al. ${ }^{7}$ found that patients with supine-predominant OSA presented a lower response to MAS treatment, but patients were treated with an appliance that allows complete mouth opening (Somnodent). Hence, differences in appliance design, specifically with respect to the potential of mouth opening, appear to be important in the context of positional OSA.,18

During sleep, mouth opening can be reduced with the use of duo-bloc devices in two different ways. The first is to choose a coupling mechanism that reduces the possibility of mandibular vertical movements. In 2010, Chung et al. ${ }^{8}$ documented that decreases in AHI and supine AHI after MAS therapy were significantly greater for the patients with positional OSA than for those with nonpositional OSA. The selected device was a TAP: a duo-bloc appliance with an anterior screw coupling mechanism that prevents mouth opening. Second, mouth opening may be controlled with anterior vertical elastics, if the coupling mechanism allows freedom of vertical movements. Recently, Norrhem and Marklund ${ }^{19}$ published a pilot study of 10 patients with moderate OSA (AHI $\geq 15$ events/h) in which they compared the outcome of MAS treatment when patients used a device (Narval, ResMed Inc) with and without vertical elastics. They did not find significant differences in AHI reduction, but two patients (20\% of the studied population) with severe OSA had a greater reduction of AHI with the use of vertical elastics. The overall nonsignificant difference may have been dependent on the fact that the device used has an inverse strip mechanism that induces increasing mandibular advancement with mouth opening. This condition could explain the higher success rate $(67 \%)$ obtained in our sample of patients treated with MAS and vertical elastics.

Our study has a number of strengths and limitations. The key strength was the use of the same MAS design in all patients, together with a similar clinical approach across both study sites. However, our study was not a randomized controlled trial and therefore we cannot exclude that differential biases across both sites had an influence on the results, although this seems unlikely. Different diagnostic methods were used at the two sites (home PG versus in-laboratory PSG) and this created a challenge in directly comparing sleep study metrics between groups. It is known that portable devices underestimate the AHI due to the 
use of total study time, rather than sleep time, as the denominator. ${ }^{13}$ To resolve this problem, we chose the proportional reduction of $\mathrm{AHI}$ as the primary outcome, allowing us to make robust comparisons between groups. Moreover, the two groups differed in some baseline factors (BMI and OSA severity) with a known influence as the treatment response. ${ }^{3}$ More specifically, patients treated with MAS and vertical elastics had more severe OSA. Despite this, we found that this group responded better to treatment. Even after statistical adjustment for age, sex, BMI, and baseline AHI we found that minimization of mouth opening improved the treatment success with respect to an equal treatment without anterior control. The findings from this retrospective study should inform the design of prospective studies to verify the importance of minimizing vertical opening in this particular OSA phenotype.

In conclusion, this retrospective study has identified a potentially important influence of MAS design on treatment outcome of patients with positional OSA. Specifically, the use of vertical elastics to prevent mouth opening appears to improve treatment outcome. These findings provide a potential explanation for the discrepant findings on the effect of MAS in positional OSA reported in the literature thus far, and support the need for further studies with a more sound methodology, such as a randomized clinical trial, to confirm our results. Within the limitation of this study, our findings highlight the potential for personalized strategies for OSA management, based on patient phenotypic characteristics.

\section{ABBREVIATIONS}

\section{AASM, American Academy of Sleep Medicine}

AHI, apnea-hypopnea index

BMI, body mass index

$\mathrm{CI}$, confidence interval

$\mathrm{IQR}$, interquartile range

MAS, mandibular advancement splint

$\mathrm{OR}$, odds ratio

OSA, obstructive sleep apnea

PG, polygraphy

PSG, polysomnography

\section{REFERENCES}

1. Cartwright RD. Effect of sleep position on sleep apnea severity. Sleep. 1984;7(2):110-114.

2. Ravesloot MJL, van Maanen JP, Dun L, de Vries N. The undervalued potential of positional therapy in position-dependent snoring and obstructive sleep apnea-a review of the literature. Sleep Breath. 2013(17):39-49.

3. Marklund M, Persson M, Franklin KA. Treatment success with an mandibular advancement device is related to supine-dependent sleep apnea. Chest. 1998;114(6):1630-1635.

4. Itasaka Y, Miyazaki S, Tada H, Ishikawa K, Togawa K. Effectiveness of prosthetic mandibular advancement for obstructive sleep apnea: analysis by sleep position. Psychiatry Clin Neurosci. 1998;52(2):225-227.

5. Yoshida K. Influence of sleep posture on response to oral appliance therapy for sleep apnea syndrome. Sleep. 2001;24(5):538-544.

6. Marklund M, Stenlund H, Franklin KA. Mandibular advancement devices in 630 men and women with obstructive sleep apnea and snoring: tolerability and predictors of treatment success. Chest. 2004;125(4):1270-1278
7. Sutherland K, Takaya H, Qian J, Petocz P, Ng AT, Cistulli PA. Oral appliance treatment response and phenotypes of obstructive sleep apnea. J Clin Sleep Med. 2015;11(8):861-868.

8. Chung JW, Enciso R, Levendowski DJ, Morgan TD, Westbrook PR, Clark GT. Treatment outcomes of mandibular advancement devices in positional and nonpositional OSA patients. Oral Surg Oral Med Oral Pathol Oral Radiol Endod. 2010;109(5):724-731.

9. Lee $\mathrm{WH}$, Wee $\mathrm{JH}$, Lee $\mathrm{CH}$, et al. Comparison between mono-bloc and bi-bloc mandibular advancement devices for obstructive sleep apnea. Eur Arch Otorhinolaryngol. 2013;270(11):2909-2913.

10. Zhou J, Liu YH. A randomised titrated crossover study comparing two oral appliances in the treatment for mild to moderate obstructive sleep apnoea/hypopnoea syndrome. J Oral Rehabil. 2012;39(12):914-922.

11. Chan AS, Cistulli PA. Oral appliance treatment of obstructive sleep apnea: an update. Curr Opin Pulm Med. 2009;15(6):591-596.

12. Iber C, Ancoli-Israel S, Chesson AL Jr, Quan SF; for the American Academy of Sleep Medicine. The AASM Manual for the Scoring of Sleep and Associated Events: Rules, Terminology and Technical Specifications. 1st ed. Westchester, IL: American Academy of Sleep Medicine; 2007.

13. Dingli K, Coleman EL, Vennelle M, et al. Evaluation of a portable device for diagnosing the sleep apnoea/hypopnea syndrome. Eur Respir J. 2003;21(2):253-259.

14. Kushida CA, Chediak A, Berry RB, et al. Clinical guidelines for the manual titration of positive airway pressure in patients with obstructive sleep apnea. J Clin Sleep Med. 2008;4(2):157-171.

15. Tagaito $\mathrm{Y}$, Isono S, Remmers JE, Tanaka A, Nishino T. Lung volume and collapsibility of the passive pharynx in patients with sleepdisordered breathing. J Appl Physiol (1985). 2007;103(4):1379-1385.

16. Ong JSL, Touyz G, Tanner S, Hillman DR, Eastwood PR, Walsh $\mathrm{JH}$. Variability of human upper airway collapsibility during sleep and the influence of body posture and sleep stage. J Sleep Res. 2011;20(4):533-537.

17. Penzel T, Moller M, Becker HF, Knaack L, Peter JH. Effect of sleep position and sleep stage on the collapsibility of the upper airways in patients with sleep apnea. Sleep. 2001;24(1):90-95.

18. Takaesu Y, Tsuiki S, Kobayashi M, Komada Y, Naayama H, Inoue Y. Mandibular advancement device as a comparable treatment to nasal continuous positive airway pressure for positional obstructive sleep apnea. J Clin Sleep Med. 2016;12(8):1113-1119.

19. Norrhem N, Marklund M. An oral appliance with or without elastic bands to control mouth opening during sleep-a randomized pilot study. Sleep Breath. 2016;20(3):929-938.

\section{SUBMISSION \& CORRESPONDENCE INFORMATION}

Submitted for publication August 4, 2017

Submitted in final revised form September 19, 2017

Accepted for publication October 5, 2017

Address correspondence to: Francesca Milano, DMD, Via Clavature 1, Bologna (BO), Italy, 40124 fr.milano@gmail.com

\section{DISCLOSURE STATEMENT}

Work for this study was performed at the University of Ferrara, Ferrara, Italy and the University of Sydney, Sydney, Australia. The Ethic Committee of Ferrara, Italy approved the research protocol the $20^{\text {th }}$ of May 2016- code: 160591. Francesca Milano is a consultant to OrthoApnea and Vivisol. Peter Cistulli holds an endowed Academic Chair position at the University of Sydney, funded by ResMed Inc. He has received research support from ResMed, SomnoMed, Zephyr Sleep Technologies, and Exploramed Inc. He is a consultant to Zephyr Sleep Technologies and NovoNordisk. He has a pecuniary interest in SomnoMed, resulting from previous involvement in research and development. The other authors report no conflicts of interest. All authors have approved the manuscript. 